\title{
Cortical rim sign and acute renal infarction
}

\author{
Po-Jen Hsiao MD, Tsung-Jui Wu MD, Shih-Hua Lin MD
}

Previously published at www.cmaj.ca

A previously healthy 42-year-old man presented to the emergency department with a two-day history of severe right flank colicky pain. He reported no history suggestive of trauma or nephrolithiasis. On physical examination, he had pain with percussion over the right costovertebral angle. Urinalysis showed 10 red blood cells and 3 leukocytes per highpower field. Laboratory investigations showed a leukocyte count of 11.7 (normal $4.5-11.0) \times 10^{\%} / \mathrm{L}$, a serum creatinine level of 115 (normal 44-88) $\mu \mathrm{mol} / \mathrm{L}$, and a serum lactate dehydrogenase level of 1462 (normal 100-250) IU/L.

Test results for prothrombin time and activated partial thromboplastin time were normal. Computed tomography (CT) of the abdomen showed nonenhancing areas over the right renal parenchyma with contrast-enhancement in the cortical area (cortical rim sign) (Figure 1A). Angiography showed several segmental thrombi in the right main renal artery (Figure 1B). Results of echocardiography were normal.

We initiated local intra-arterial thrombolytic therapy with urokinase, followed by systemic anticoagulation with heparin. On his fourth day in hospital, the patient's serum creatinine level was $80 \mu \mathrm{mol} / \mathrm{L}$ and levels of plasma protein $\mathrm{C}$, protein $\mathrm{S}$, and antithrombin III were normal. Based on the lack of identifiable causes, we diagnosed idiopathic acute renal infarction. The patient was given warfarin. At one-year follow-up, he had not had a recurrence.

Acute renal infarction can be cardiogenic or noncardiogenic in origin. In patients with noncardiogenic acute renal infarction who have no apparent risk factors (e.g., thromboembolism, coagulation dysfunction, hematologic diseases) the cause often remains elusive. These instances are labelled as idiopathic, as in our patient. ${ }^{1,2}$ The diagnosis of acute renal infarction is often delayed because of a low index of suspi-

From the Division of Nephrology, Department of Medicine, Tri-Service Genera Hospital, National Defense Medical Center, Taipei, Taiwan, Republic of China

\section{CMAJ 2010. DOI:10.1503/cmaj.091110}

cion by physicians. Clinical features include unexplained acute pain in the flank or abdomen, and elevated serum lactate dehydrogenase levels, even in the absence of heart disease. Contrast-enhanced CT helps to facilitate a rapid diagnosis. The cortical rim sign, seen in about $50 \%$ of acute renal infarctions, ${ }^{3}$ is relatively specific to this condition and believed to result from intact renal collateral circulation following a vascular insult, such as renal artery thrombosis or embolization, renal artery dissection or renal trauma. Therapeutic guidelines for acute renal infarction are not well established. Anticoagulation therapy may be as effective as surgical intervention. ${ }^{1,2}$ Given the limited viability of the unperfused kidney, a successful intervention relies on prompt diagnosis.

This article has been peer reviewed.

Competing interests: None declared.

\section{REFERENCES}

1. Huang CC, Lo HC, Huang $\mathrm{HH}$, et al. ED presentations of acute renal infarction. Am J Emerg Med 2007;25:164-9.

2. Bolderman R, Oyen R, Verrijcken A, et al. Idiopathic renal infarction. Am J Med 2006;119:356.e9-12.

3. Regine G, Stasolla A, Miele V. Multidetector computed tomography of the renal arteries in vascular emergencies. Eur J Radiol 2007;64:83-91. 